\title{
Streptobacillosis characterised by palmoplantar pustulosis
}

Lancet Infect Dis 2013; 13: 96 Hôpitaux Universitaires de Strasbourg, Department of Internal Medecine, Hôpital de

Hautepierre (F Danion MD, E Bui MD, B Goichot MD), and Department of Bacteriology, Hôpital Civil (Prof P Riegel PhD)

Université de Strasbourg,

Strasbourg, France

Correspondence to:

Mr Francois Danion, Department of Internal Medicine, Hôpitaux Universitaires de Strasbourg, 67098 Strasbourg, France francois.danion@chrustrasbourg.fr
Francois Danion, Erwan Bui, Philippe Riegel, Bernard Goichot

A 41-year-old French woman, with no previous medical history, presented with intermittent fever up to $40^{\circ} \mathrm{C}$. 2 days later, palmoplantar pustules appeared (figure, A), as did cutaneous papules on her trunk and limbs. She had an oligoarthritis associated with the distal interphalangeal joint of the third left finger, the right knee, and both shoulders. The patient maintained that she was in good health until she was bitten by a domestic rat 1 week before the symptoms began. She did not report engaging in unsafe sexual activity.

A cutaneous biopsy showed a leucocytoclastic vasculitis. The transthoracic and oesophageal echocardiography, and the CT scan were normal. Blood cultures were positive for a Gram-negative bacillus; the bacterial cells were arranged in chains and clumps with lateral bulbar swellings, which is highly consistent with streptobacillosis (figure, B). The sequencing of the 16S rRNA gene identified Streptobacillus moniliformis. The patient was treated with amoxicillin at $6 \mathrm{~g}$ per day for 6 weeks, because of arthritis. The symptoms subsequently disappeared and the patient recovered.

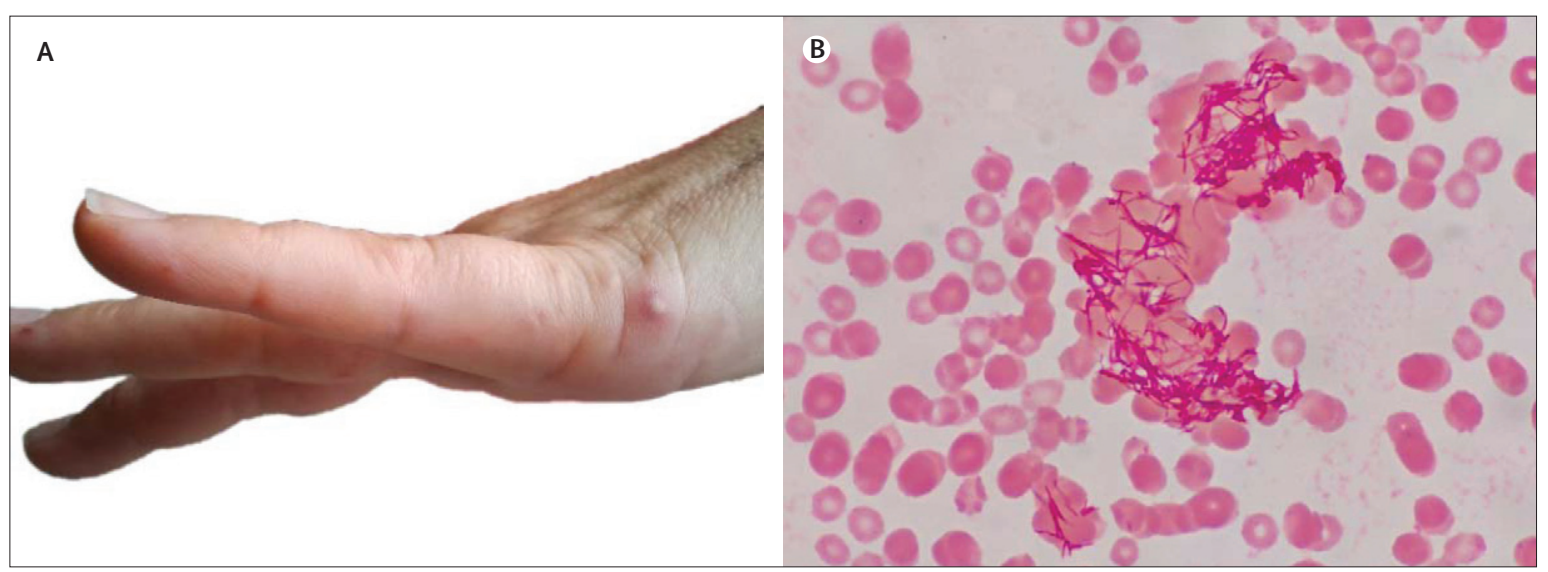

6 months after the infection, she was well and had no medical complaints.

Streptobacillosis-also known as rat-bite fever-is caused by $S$ moniliformis. This Gram-negative bacillus is part of the oropharyngeal commensal flora of the rat and is transmitted to human beings by rat bites. The disease is characterised by fever, cutaneous rash, and migratory polyarthralgia. Without treatment, the mortality rate is about $13 \%$. A palmoplantar pustulosis suggests a diagnosis, such as endocarditis, gonorrhoea, coxsackie virus, SAPHO (synovitis, acne, pustulosis, hyperostosis, osteitis) syndrome-a chronic inflammatory osteoarticular disease associated with skin abnormalities and pustular psoriasis. However, palmoplantar pustulosis is an usual sign of streptobacillosis, which is rarely mentioned as a differential diagnosis of pustulosis. Attention should be paid to the increased incidence of this disease, because pets (especially rats and mice), are the most important vector of this disease.

Conflicts of interest

We declare that we have no conflicts of interest. 